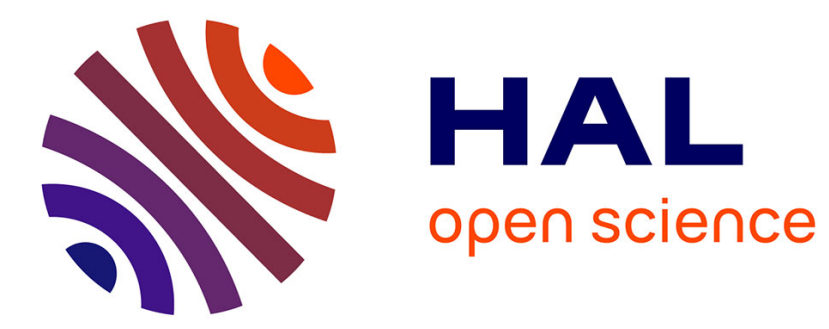

\title{
Phylogeny of Embodied Evolutionary Robotics
}

Amine Boumaza

\section{To cite this version:}

Amine Boumaza. Phylogeny of Embodied Evolutionary Robotics. Second Workshop on Evolving Collective Behaviors in Robotics at GECCO'17, Jul 2017, Berlin, Germany. pp.1681-1682 10.1145/3067695.3082547. hal-01516044

\section{HAL Id: hal-01516044 \\ https://hal.inria.fr/hal-01516044}

Submitted on 28 Apr 2017

HAL is a multi-disciplinary open access archive for the deposit and dissemination of scientific research documents, whether they are published or not. The documents may come from teaching and research institutions in France or abroad, or from public or private research centers.
L'archive ouverte pluridisciplinaire HAL, est destinée au dépôt et à la diffusion de documents scientifiques de niveau recherche, publiés ou non, émanant des établissements d'enseignement et de recherche français ou étrangers, des laboratoires publics ou privés. 


\title{
Phylogeny of Embodied Evolutionary Robotics
}

\author{
Amine Boumaza \\ Université de Lorraine, LORIA \\ Campus scientifique BP 239 \\ Vandoeuvre-lès-Nancy Cedex, F-54506, France \\ amine.boumaza@loria.fr \\ Initial draft: April 6th, 2017 \\ Updated: April 28, 2017
}

\section{Introduction}

Embodied evolutionary robotics (EER), aims to learn collective behaviors for a swarms of agents, where evolution is distributed on the agents that adapt online to the task [4]. In this context, mEDEA (minimal Environment-driven Distributed Evolutionary Algorithm) [1] adapts a swarm of robots which exchange genetic material when they meet. In its original form, the algorithm does not push toward a task driven fitness but rather takes an open-ended view in which genes survive by spreading in the swarm. Successful genes whose copies survive throughout many generations must: 1) maximize mating opportunities and 2) minimize the risk for their vehicles. We propose to use phylogenetic trees that record the dissemination of the genomes and study their structure. Our motivation in doing so is to provide a new angle from which we can study these algorithms that may be complementary to traditional tools.

We consider the original version of mEDEA augmented by a task driven fitness and to which we add two slight modifications (Algorithm 1): on the one hand, the agent's genome is always in its list (we call this self-insemination), and on the other hand, a selection pressure using a tournament with adjustable size. Self-insemination prevents from having inactive agents that were unsuccessful at gathering new genetic material whereas the adjustable tournament allows to tune the level of selection pressure.

\section{EER phylogeny}

We record the dissemination of the genes between agents and track their descendants to construct a phylogenetic tree. Nodes in the tree represent active genomes in the swarm and edges represent parenthood relations (child nodes are one mu- 


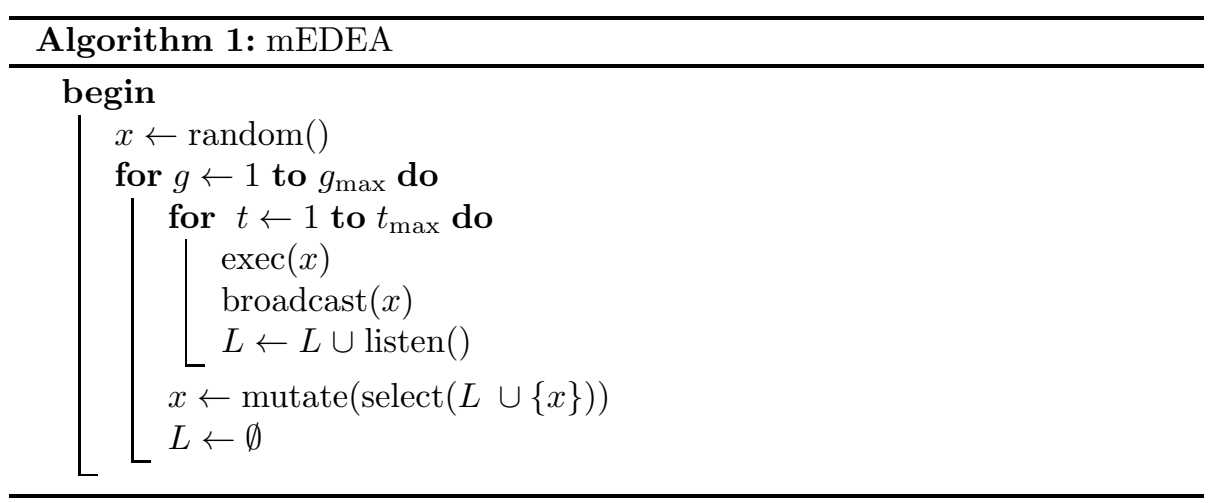

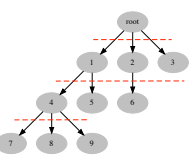

(a) Simple tree.

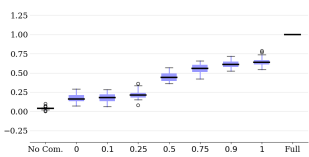

(d) $S F$

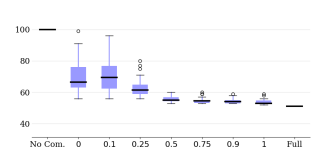

(b) Tree radius.

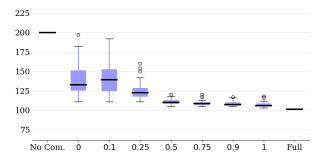

(c) Tree diameter.

Figure 1: The effect to selection pressure on different measures.

tation away from their parents). We artificially add a "root" node, parent of all initial random genes ${ }^{1}$. Each node, that is neither the root nor an initial genome, has at most one parent, and can have at most $n$ children, $n$ being the size of the swarm. Finally, the depth of the tree is at most $g_{\max }$, and at each level there are exactly $n$ nodes (this is due to self-insemination). A simplified phylogenetic tree is shown in Figure 1 (a).

Let $\mathcal{T}=(\mathcal{V}, \mathcal{E})$ be a phylogenetic tree created by an execution of Algorithm 1 where $\mathcal{V}$ are vertices and $\mathcal{E}$ are edges. We will also use $\mathcal{V}^{d}$ to note the nodes at depth $d$ and $\|p(u, v)\|$ the length of the path, when it exists, between nodes $u$ and $v$. Let $\operatorname{deg}(u)$ be the degree of node $u$ (the number of its offspring) and $f(u)$ the fitness of genome $u$.

We define the survival rate at depth $d$ as the proportion of initial genomes that have offspring at depth $d$ or more formally:

$$
S r^{d}=\frac{1}{n} \sum_{r \in \mathcal{V}^{0}} P^{d}(r)
$$

where

$$
P^{d}(u)=\left\{\begin{array}{l}
1 \text { if } \exists v \in \mathcal{V}^{d} \mid\|p(u, v)\|>0 \\
0 \text { otherwise. }
\end{array}\right.
$$

\footnotetext{
${ }^{1}$ This trick allows to have a connected graph to ease the analysis and does not change the results.
} 
Let $S g^{d}=\arg \max _{u \in \mathcal{V}^{d}} \operatorname{deg}(u)$ be the genome with the most offspring at generation $d$ and similarly $F g^{d}=\arg \max _{u \in \mathcal{V}^{d}} f(u)$ the fittest. Furthermore, let

$$
S F=\frac{1}{g_{\max }} \sum_{d=1}^{g_{\max }} I\left(S g^{d}, F g^{d}\right)
$$

where

$$
I(u, d)=\left\{\begin{array}{l}
1 \text { if } u=v \\
0 \text { otherwise. }
\end{array}\right.
$$

and

$$
S F_{r}=\left[\frac{1}{g_{\max }} \sum_{d=1}^{g_{\max }}\left(f\left(S g^{d}\right)-f\left(F g^{d}\right)\right)^{2}\right]^{1 / 2}
$$

$S F$ measures how many times the genome with the most offspring is the one with the highest fitness and $S F_{r}$ measures the difference in their fitness values.

We are interested in the following questions : 1) how long does it take for the lineage of the initial genomes to be extinct ? 2) Is the fittest gene the one that has the most offspring? How these values behave when we apply different levels of selection pressure?

The experiments were performed on the Roborobo simulator [2], on a obstacle free circular environment of size $1000 \times 1000$ with $n=200$ agents. Genomes code the weights of a perceptron (12 proximity sensors, and two wheel velocities). We fix $t_{\max }=200$ and $g_{\max }=100$. The task we address is navigation and obstacle avoidance and we use the well known fitness defined in [3]. Mutation strength was fixed at $\sigma=0.25$.

The results presented in Figure 1 (b-e) are aggregated on 30 independent runs with different selection pressures (noted on the abscissa axis) ranging from 0 for random to 1 for elitist. We added two sets of runs that correspond to unrealistic extreme cases: where no communication is allowed between agents (No com.), and where every agents exchange with all other agents (Full broadcast) and selection is elitist.

If we examine the structures of the trees in term of degree and radius, we see that both values decrease when selection pressure increase. Trees are more balanced and somewhat "fatter" when selection pressure is low than when it is high where they are slimmer and more elongated which suggests less lineages. If we look at the distribution of $S F$ and $S F_{r}$ Figure 1 (d, e), we can see that for high levels of selection pressure the fittest genes tend to have the most offspring. We should although, note that when no selection is performed almost one in four generations the fittest creates the most offspring ! Open-ended evolution tends to favor fit individuals. Finally, looking at the survival rate Figure 1(f), we note that in all situations lineages of the initial genes tend to disappear relatively quickly depending on the pressure, all genomes at the end of the evolution descend from one gene of the initial parents. 


\section{Conclusions}

In this paper we explore the idea of analyzing EER from the perspective of genes and their dynamics using phylogenetic trees. We believe that taking this perspective could provide interesting insights. We addressed a simple question regarding the dynamics of the fittest and most copied genes as an illustration, but there are several open questions worth investigating: what is the structure of the trees produced, do different executions share similar tree structures ? Can we infer properties on the execution from the structures of the tree? Could we use tools from spectral graph theory or computational phylogenetics to study the behavior of EER algorithms?

\section{References}

[1] Nicolas Bredeche and Jean-Marc Montanier. Environment-driven Embodied Evolution in a Population of Autonomous Agents. In PPSN 2010, pages 290-299, Krakow, Poland, 2010.

[2] Nicolas Bredeche, Jean-Marc Montanier, Berend Weel, and Evert Haasdijk. Roborobo! a fast robot simulator for swarm and collective robotics. CoRR, abs/1304.2888, 2013.

[3] Stefano Nolfi and Dario Floreano. Evolutionary Robotics: The Biology, Intelligence, and Technology. MIT Press, Cambridge, MA, USA, 2000.

[4] Richard A. Watson, Sevan G. Ficici, and Jordan B. Pollack. Embodied evolution: Distributing an evolutionary algorithm in a population of robots. Robotics and Autonomous Systems, 39:1-18, 2002. 